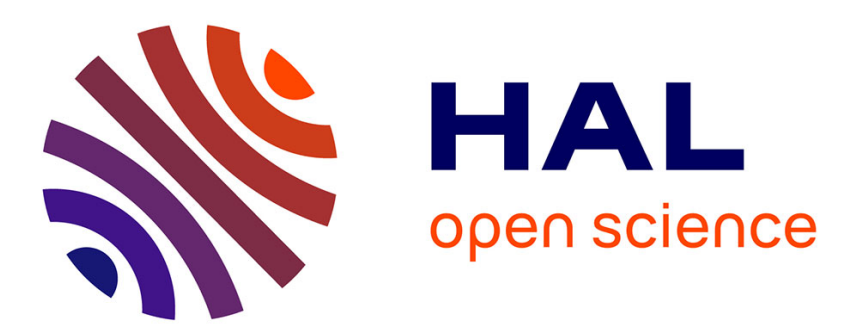

\title{
Experiments and micromechanical modelling of elastic parameters of a composite geomaterial
}

\author{
Jolanta Lewandowska, Maciej Pilawski
}

\section{To cite this version:}

Jolanta Lewandowska, Maciej Pilawski. Experiments and micromechanical modelling of elastic parameters of a composite geomaterial. European Journal of Environmental and Civil Engineering, 2012, 17 (1), pp.46-63. 10.1080/19648189.2012.667694 . hal-00826190

\section{HAL Id: hal-00826190 https://hal.science/hal-00826190}

Submitted on 27 May 2013

HAL is a multi-disciplinary open access archive for the deposit and dissemination of scientific research documents, whether they are published or not. The documents may come from teaching and research institutions in France or abroad, or from public or private research centers.
L'archive ouverte pluridisciplinaire HAL, est destinée au dépôt et à la diffusion de documents scientifiques de niveau recherche, publiés ou non, émanant des établissements d'enseignement et de recherche français ou étrangers, des laboratoires publics ou privés. 


\title{
Experiments and micromechanical modelling of elastic parameters of a composite geomaterial
}

\author{
Jolanta Lewandowska**** — Maciej Pilawski*** \\ * Laboratoire de Mécanique et Génie Civil de Montpellier (LMGC), UMR 5508 \\ CNRS, Université Montpellier 2 \\ CC 048 Place Eugène Bataillon \\ 34095 Montpellier cedex 5 \\ Jolanta.Lewandowska@univ-montp2.fr \\ **U JF-Grenoble 1 / Grenoble-INP / CNRS UMR 5521, \\ Laboratoire 3SR Grenoble, F-38041, France (formerly) \\ *** Silesian University of Technology \\ Ul. Akademicka 5 \\ 44-100 Gliwice \\ pilawski.maciej@gmail.com
}

\begin{abstract}
The aim of this paper is to study the elastic behaviour of a periodic composite geomaterial by applying a micromechanical approach. The macroscopic anisotropic rigidity tensor is computed using the asymptotic expansion homogenization method. The results are partially validated through the triaxial testing of a composite in which the spheres made of sintered clay are periodically embedded in a matrix (Hostun sand HN38). Good agreement was observed between calculated and measured elastic parameters.

RÉSUMÉ. L'objectif de cet article est d'étudier le comportement élastique d'un géomatériau composite périodique en appliquant l'approche micromécanique. Le tenseur de rigidité macroscopique anisotrope a été calculé en utilisant la méthode des développements asymptotiques. Les résultats ont été partiellement validés par les essais triaxiaux sur un composite constitué des sphères d'argile solidifiée réparties périodiquement dans la matrice (sable d'Hostun HN38). La comparaison montre un bon accord entre les paramètres élastiques calculés et mesurés.

KEYWORDS: Homogenization - Rigidity tensor - Triaxial tests - Geomaterials - Numerical computations - Anisotropy
\end{abstract}

Revue. Volume $\mathrm{X}-\mathrm{n}^{\circ} \mathrm{x} /$ année, pages 1 à $\mathrm{X}$ 


\section{Introduction}

The elastic behaviour of composite geomaterials is of interest because of potential applications of such materials in environmental geomechanics. In particular, many ideas have been recently developed concerning the so called "green geotechnics", that focuses on the re-utilisation of waste materials like for example the coal combustion products in civil engineering and road making. The use of tire shreds in construction projects, such as highway embankments, is also becoming one of the commonly accepted ways of beneficially recycling scrap tires. Although the general idea of recycling the industrial wastes is not new, there is a new context for such activity due to recent regulatory initiatives at the European level (see for example REACH available on the web).

The literature concerning the effective elastic properties of heterogeneous materials is very rich. There exist many different methods, using classical to modern approaches based on micromechanical considerations. The very comprehensive review of the state of the art can be found for example in the books by Bornert et al. (2001), Torquato (2005), François et al. (2009) and Suquet (1997). The most classical approach consisted in the prediction of limits (upper and lower) for the effective parameters such as effective bulk and shear modulus. The commonly known limits based on the volumetric fractions and the elastic parameters of the components were proposed by Voigt (1887) - Reuss (1929) and Hashin - Shtrikman (1962, 1963). Although they can be useful in some cases, the general practical applicability of the method of bounds is very limited.

It is evident that more precise prediction requires more information about the internal structure of the material, which is now accessible thanks to the recent development of X-ray microtomography and the image correlation techniques (computed tomography) (Hall et al., 2010), (Lenoir et al., 2007).

The microstructural information can be provided in a statistical sense or in a deterministic (explicit) manner. Some of the modern methods use the approach of estimations to construct a big number of solutions for some morphological classes of materials (see for example in François et al. (2009). In this case the morphological information is given through the assumption about the form and the spatial distribution of inclusions. In Torquato (2005) a systematic theory of random heterogeneous materials is proposed. "The details of the microstructure" like the phase volume fractions, surface area of interfaces, orientations, sizes, shapes and spatial distribution the phase domains or connectivity of the phases are approached statistically by n-point correlation functions. A large number of solutions for particular cases were developed.

Another approach concerns the media with periodic microstructure. In this case the microstructure of the medium is given explicitly, which is very important when modelling the coupled transient phenomena taking place in heterogeneous porous materials. For example, the knowledge of connectivity of phases is essential in 
modelling of chemo-mechanical couplings. Qualitatively different macroscopic models (with different effective parameters) should be applied to model mass transfer, in case of fractured material when the network of fractures is connected or not.

The asymptotic expansion homogenization method (called also periodic homogenization), dealing with periodic structures, was firstly introduced by (Sanchez-Palencia, 1980) and (Bensoussan et al., 1978). Since then, it has been widely used for modelling of different periodic composite materials (see for example the numerical computations in Bornert at al. (2001) or homogenization of a special case recently presented in Chatzigeorgiou et al., 2011, among others). The homogenization makes it possible to link the microstructure and the elastic parameters of the components with the macroscopic behaviour of the composite system when the size of the heterogeneity with respect to the macroscopic length is small. Thus, it is possible to construct materials of desired properties for specific engineering applications.

The aim of the paper is to present the application of the upscaling method by using the asymptotic expansions (Sanchez-Palencia, 1980) and (Bensoussan et al., 1978) for the prediction of the macroscopic elasticity parameters of the three dimensional anisotropic composite. It is shown how the asymptotic expansion homogenization method can be used in a particular case of periodic porous composite made of sand and solidified clay spheres (double porosity medium). In addition, the results obtained by numerical calculations of the macroscopic rigidity tensor were verified experimentally by performing a series of triaxial tests in consolidated drained conditions (CD triaxial compression tests).

The paper is structured as follows. In Section 2 we describe the composite material and the experimental investigations of this material in the triaxial apparatus. The homogenization analysis of the elasticity problem for the double porosity composite is discussed in section 3. Section 4 presents the numerical calculations of the rigidity tensor, and the interpretation and comparison with the experimental data. In the last section 5 the final conclusions are formulated.

\section{Double porosity composite material and triaxial testing}

The double porosity composite material investigated in this paper was build from two components: Hostun fine sand and small spheres made of solidified clay embedded periodically in the sand. This material was conceived for modelling purposes, in particular for experimental verification of theoretical and numerical modelling of macroscopic multiphysical behaviour by using the multi-scale homogenization approach (Lewandowska et al. 2005, 2008), (Szymkiewicz et al., 2008), (Tran Ngoc at al., 2011). 
Several mechanical tests were performed in the triaxial apparatus in order to determine the elastic parameters of the composite geomaterial (Grinke, 2007). The same tests were also carried out in pure sand to obtain the characteristics of matrix component of the composite, that is a necessary input data for numerical computations. Moreover, the inter-comparison of the triaxial curves enabled us to experimentally observe the influence of the second component (solidified clay) on the behaviour of the whole system.

\subsection{Double porosity composite material}

The two components i.e. the homogenous Hostun sand HN38 and the solidified clayey spheres were used to build a composite in a triaxial cylinder of the diameter of $10 \mathrm{~cm}$ and the height of $10 \mathrm{~cm}$. The cylinder was filled up alternatively with sand and spheres (layer by layer) by controlling the mass and the mechanical energy of compaction. We proceeded in the following way: i) a layer of a given quantity of spheres was carefully put into the cylinder, so that the spheres touched each other in the horizontal plane, ii) a layer of a given mass of sand was put on the top of the spheres in order to cover the spheres, iii) then both layers were compacted by using a small hammer. This procedure was continued until the cylinder was filled. The volumetric fractions of each component material were $50 \%$ and $50 \%$, respectively. The established and rigorously respected experimental protocol (Grinke, 2007) enabled us to obtain the composite material of periodic structure of the cubic-centered type (but not exactly the cubic-centered), Figure 1. More details about the microstructure can be found in section 3.2.

The laser granulometry of the sand HN38 showed a uniform grain size distribution with the mean grain size of $162 \mu \mathrm{m}$. The mean diameter of the clay spheres was $6.4 \mathrm{~mm}$. The mercury porosity test gave the porosity of spheres $\phi=$ 0.376 and the mean pore size around $0.7 \mu \mathrm{m}$. The skeleton specific density of the spheres material was $\rho_{\mathrm{s}}=3.01 \mathrm{~g} / \mathrm{cm}_{3}$ and the dry bulk density $\rho_{\mathrm{d}}=1.88 \mathrm{~g} / \mathrm{cm}_{3}$. The porosity of the homogeneous sand samples and the porosity of sand used in the composite material samples were controlled and kept constant in both cases, $\mathrm{n}=$ 0.40. Prior to mechanical testing both materials were subjected to different kind of tests for other modelling purposes, so their physical properties are well known (Lewandowska et al. 2005, 2008), (Szymkiewicz et al., 2008), (Tran Ngoc at al., 2011).

The triaxial tests were perfomed at the Laboratory 3S-R in Grenoble, France. The apparatus was manufactured by Wykeham Farrance. It was equipped with a load cell of the capacity $100 \mathrm{kN}$. The variations of volume of the sample were measured by a manometric volume gauge. We followed the procedure of $\mathrm{CD}$ triaxial testing of granular materials established at the Laboratory 3S-R (Desrues and Orestis, 2006). The precision of measurements were: $26 \mathrm{~N}$ for force and $0.08 \%$ for volume change. The details concerning the tests presented in this paper can be found in (Grinke, 2007) and (Pilawski, 2011). 


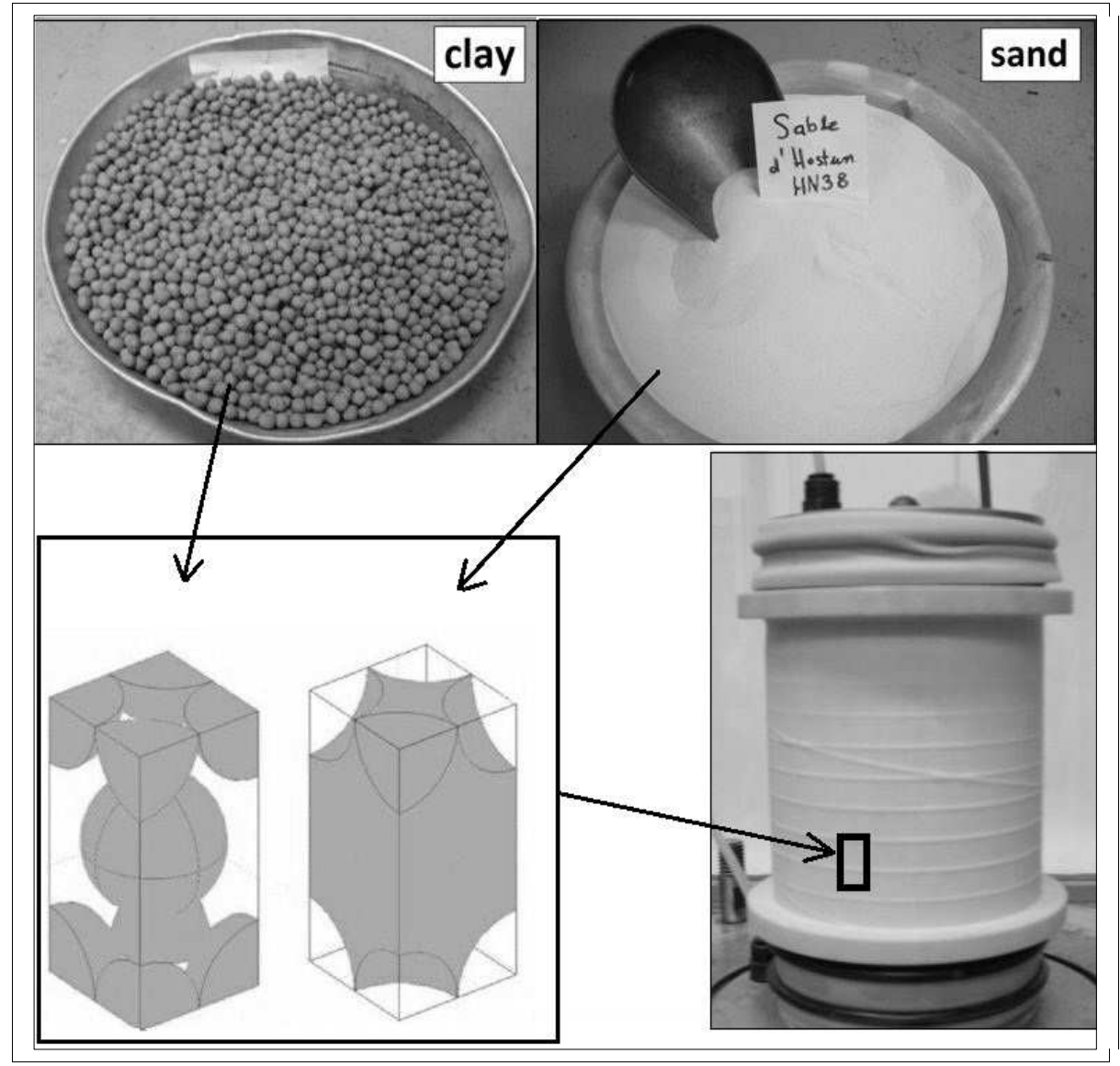

Figure 1. Periodic structure of the composite (Grinke, 2007)

\subsection{Experimental program}

In order to determine the elastic parameters a series of classical triaxial tests in the composite and in the pure sand, was performed. All samples were initially saturated by water using the back pressure technique $(100 \mathrm{kPa})$. The values of Bishop coefficient were relatively high and varied between $\mathrm{B}=82 \%$ and $\mathrm{B}=90 \%$. The speed of the axial loading displacement device in strain controlled conditions was $0.12 \mathrm{~mm} / \mathrm{min}$.

Two kinds of triaxial tests were performed:

- $\quad$ the CD shearing test under constant confining pressure of $100 \mathrm{kPa}$

Revue. Volume $\mathrm{X}-\mathrm{n}^{\circ} \mathrm{x} /$ année, pages 1 à $\mathrm{X}$ 
- the isotropic compression test in drained conditions; the range of pressure was $50 \mathrm{kPa}-400 \mathrm{kPa}$. The pressure was increased by steps of $50 \mathrm{kPa}$

During all tests the volume changes were registered by measuring the volume of water drained out of the sample. The shearing tests continued until $10 \%$ of axial deformation was reached. During each shearing experiment three unloadings were done, after 7, 14 and 21 minutes from the beginning of the test respectively. Thus, we restricted the deformation zone to max $2 \%$. Both materials were tested in the same conditions. Note that this composite material of periodic microstructure was studied under conditions of scale separation. The scale separation means the ratio of the dimension of the period over the dimension of the macroscopic domain (here the size of the sample).

\subsection{Results}

\subsubsection{Comparison of the triaxial curves}

In Fig. 2 and Fig. 3 the results of shearing tests in sand and in the composite, are presented. In Fig 2 the curve of axial stresc ac a function of axial strain, whereas in Fig 3 the curve of relative volume change ( $\Delta V / V_{0}$ where $V_{0}$ is the initial volume) as a function of axial strain, are plotted. By comparing the curves for the two materials we can observe the qualitative difference in the mechanical behaviour of both materials, from rather ductile (for sand) to rather fragile (for composite) type of behaviour.

In Fig. 4 an example of unloading during triaxial test in the double porosity medium (composite) in the strain range: 0.0183 - 0.0194, is shown. The interpretation of these results is presented in section 2.3.2. In Fig. 5 we can see the relative volume changes with time corresponding to each isotropic stress increment for both materials. It can be seen that the volume changes in case of composite are considerably less than in case of sand. Moreover, some irregularities in the curve for composite are observed (see section 4.3 for discussion of this effect). 


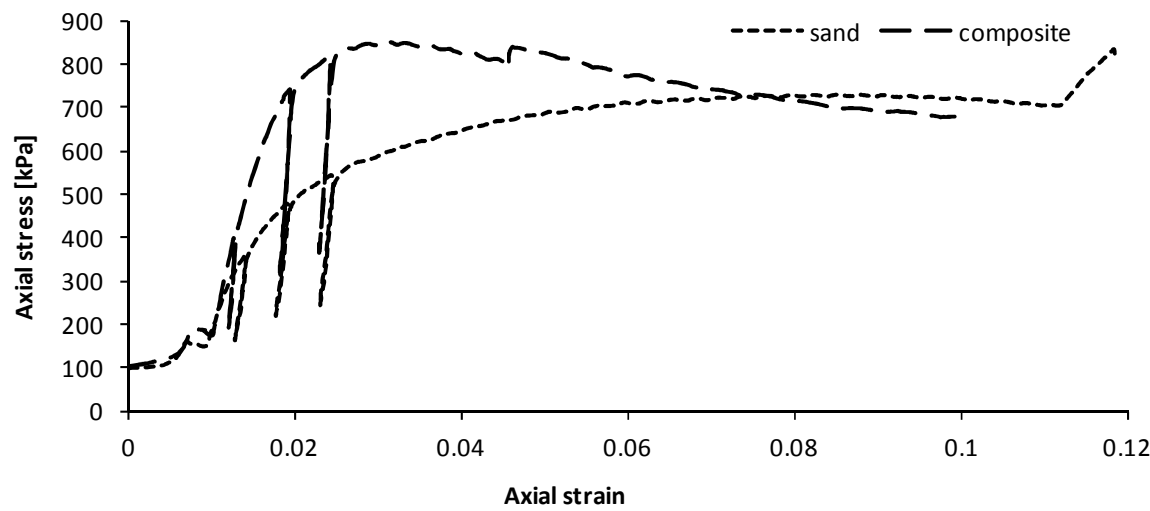

Figure 2. Axial stress as a function of axial strain

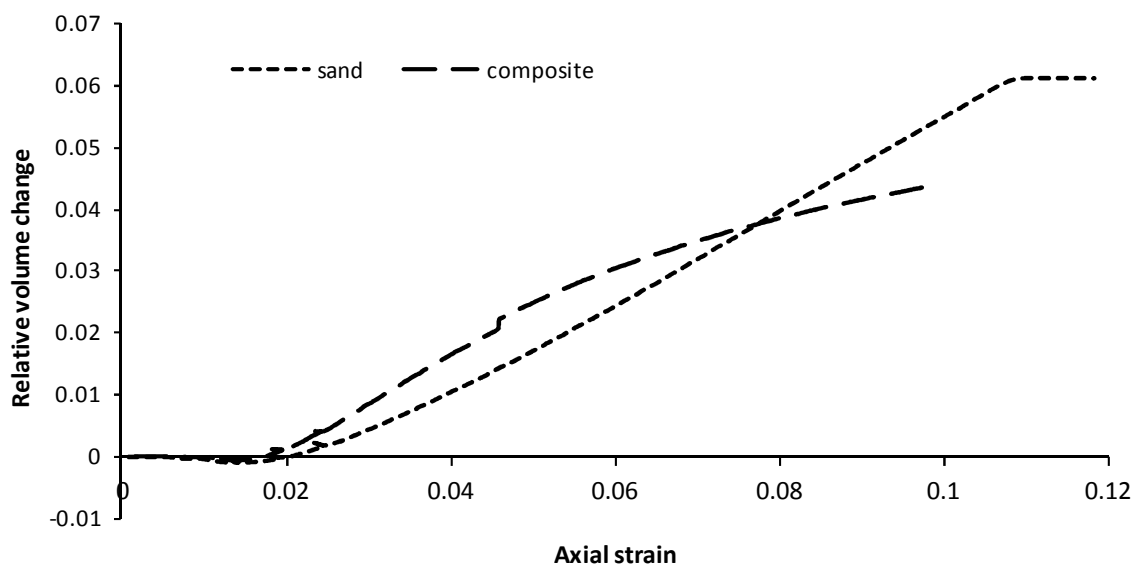

Figure 3. Relative volume change as a function of axial strain 
8 Revue. Volume $\mathrm{X}-\mathrm{n}^{\circ} \mathrm{x} /$ année
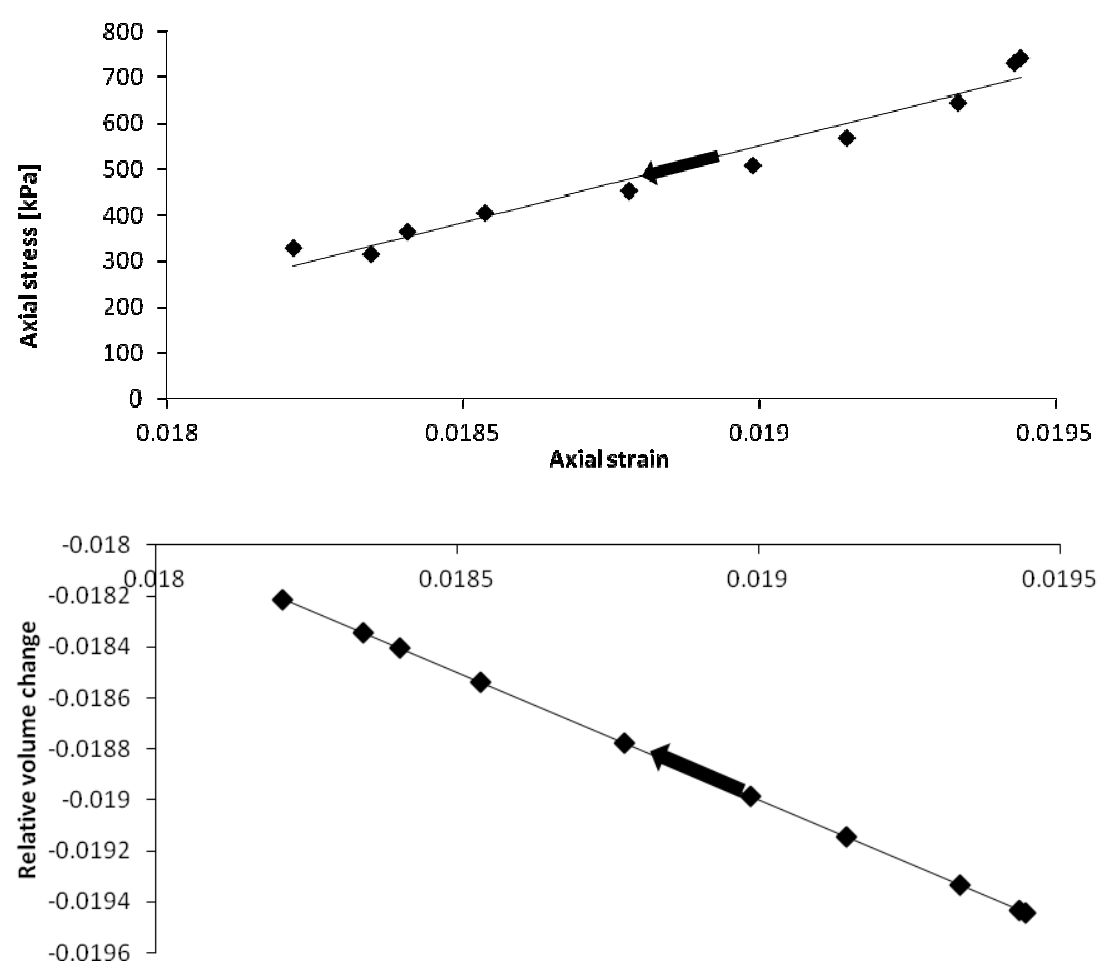

Figure 4. Example of the unloading during triaxial test in the double porosity medium (composite) in the strain range: $0.0183-0.0194$. 
Titre courant de l'article $\quad 9$

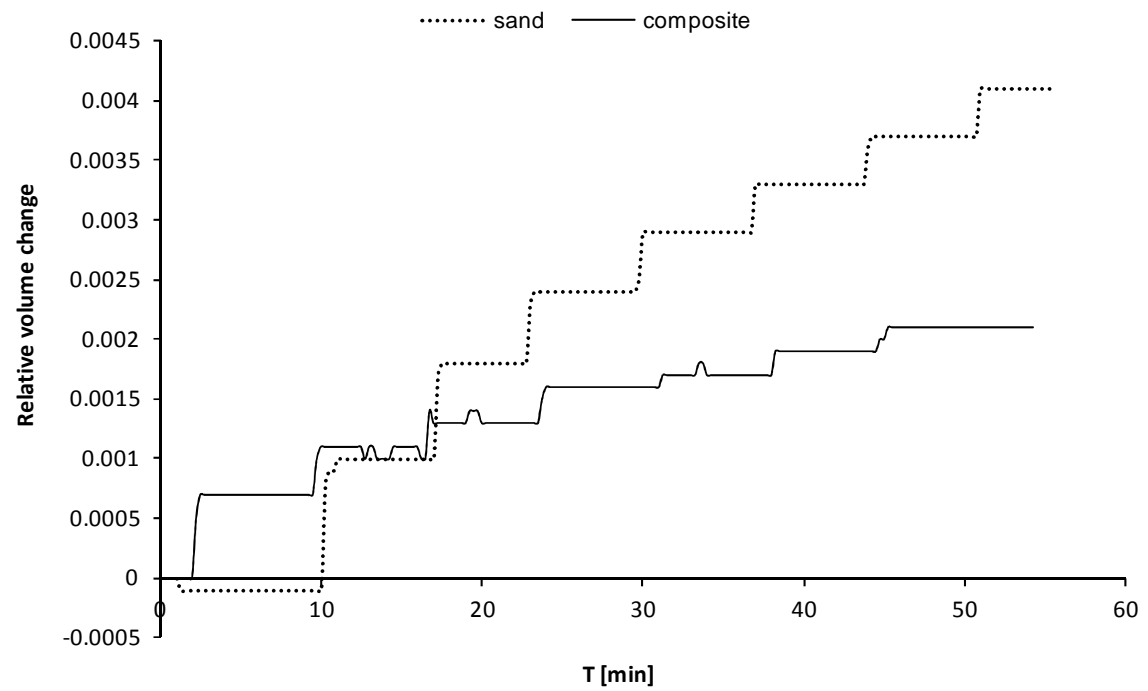

Figure 5. Relative volume change as a function of time during isotropic compression test 


\subsubsection{Interpretation of the results of the triaxial tests}

The obtained results were interpreted in the framework of the theory of elasticity. From three unloadings parts of the triaxial shearing curves in sand and in the composite we calculated the Young modulus in the axial (vertical) direction, and the Poisson ratio in both lateral directions (corresponding to the axial loading). On another hand, a bulk modulus $\mathrm{K}$ was calculated from the isotropic compression tests results for each material. Two tests in sand (Test 1 and Test 2) were used. The results of all calculations are presented in Tab. 1.

It can be noticed that the elastic parameters of both materials depend on the range of the strains and stresses. For sand a clear increase of the Young modulus is seen. For the composite the increase is less evident. Moreover, the presence of solidified clay inclusions leads to substantial increase of elastic parameters of the composite with respect to the pure sand: 2.36 times for the Young modulus, 1.16 times for the Poisson ratio and the 1.78 times for the bulk modulus. Finally, the Poisson ratio of the composite seems to be rather unrealistically high (close to 0.5), especially if we compare it with the value obtained for pure sand (the matrix in the composite). It will be further discussed in section 4.3 . 
Table 1. Triaxial tests results

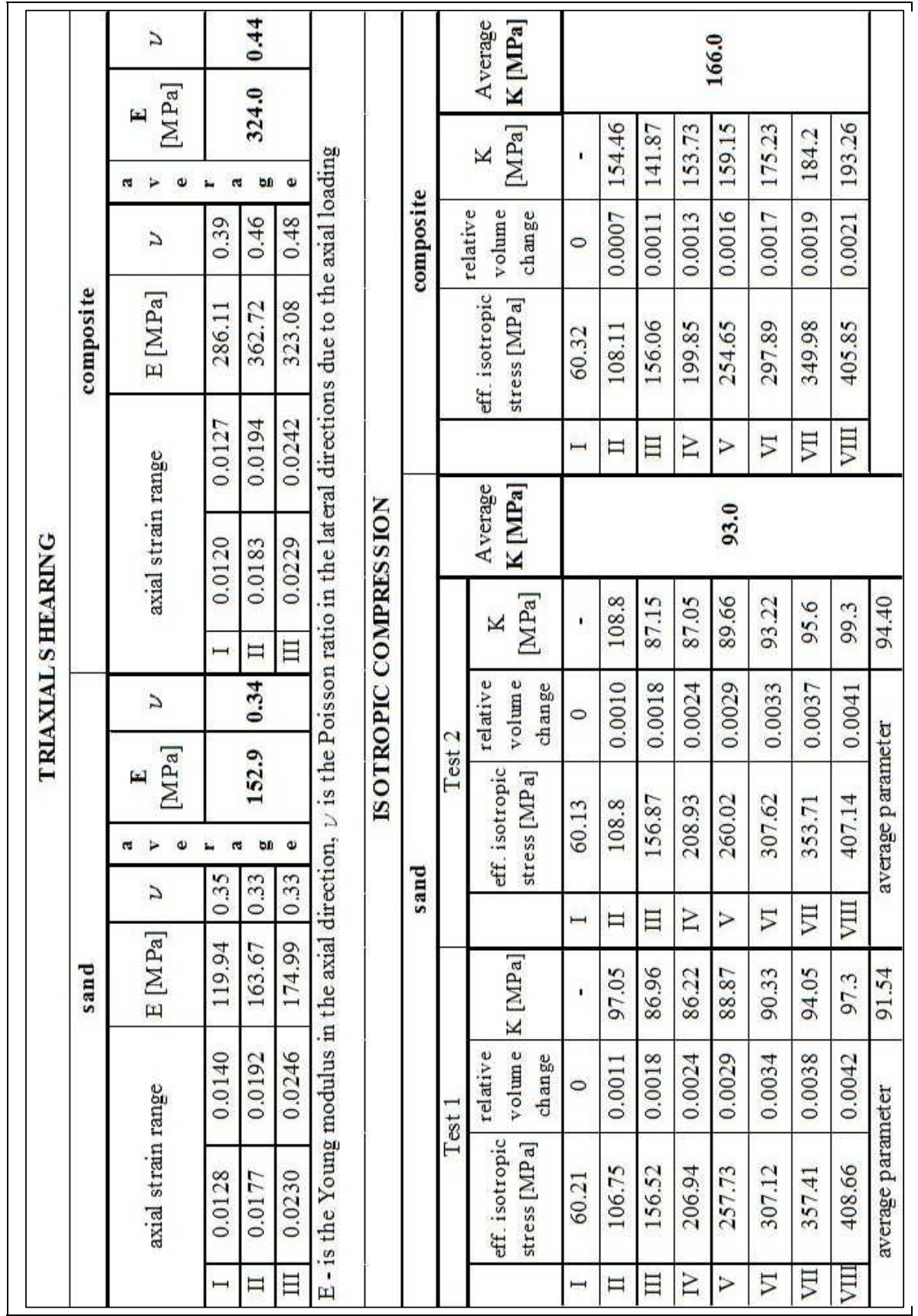




\section{Homogenization analysis of the double porosity composite material}

\subsection{Multiscale approach}

In order to obtain the effective elastic properties of the double porosity composite the asymptotic expansion homogenization method was used. The method is well established in the literature (Sanchez-Palencia, 1980; Bensoussan et al. 1978). In its classical form the method concerns two scales, the microscopic and the macroscopic sale. The double porosity composite material analyzed in this paper is considered as a three scale material, with micro, meso and macroscopic scales. Therefore, to carry out the homogenization we have to start from the smallest scale (the pore scale) by freezing all larger scales. Once the homogenization is done at this scale, the material properties and the corresponding physical description can be shifted to the next scale, and the process starts again. The required condition for such a multiscale approach to be possible is the scale separation at each scale level (Auriault, 1991).

In the paper it is considered that the first homogenization process has been already done (Sanchez-Palencia, 1980), (Bensoussan et al. 1978), and we focus on the second step of homogenization. For simplicity of the presentation in the following development the notations "micro" and "macro" will be used for the second step of homogenization

\subsection{Microstructure and microscopic properties}

The choice of the three dimensional microstructure analysed in this paper was motivated by the interest of dealing with a composite material presenting macroscopic anisotropy. Moreover, the inclusions are interacting in the horizontal directions and the material of inclusions is compressible. For the composite material of such microstructure the experimental data presented in section 2 are available. Thus, the comparison between computations and measurements will be possible.

Three layers of spheres embedded in a matrix material, can be seen in Figure 6. The spheres touch each other in each horizontal layer and form cubic-like arrangements in the horizontal and vertical planes. Taking into account the whole three dimensional structure, the arrangement can be called of "centered-cubic" like. The external dimensions of the period are: $6.4 \mathrm{~mm} \times 6.4 \mathrm{~mm} \times 13.404 \mathrm{~mm}$. 


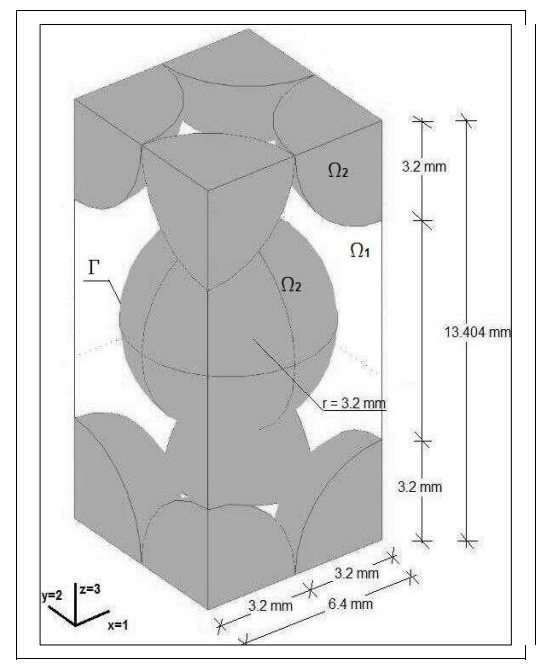

Figure 6 Geometry of the periodic cell

The first process of homogenization (not presented here) concerns the constituents of the composite i.e. sand and sintered clay, taken individually. We assume that the after the first step of homogenization both components of the composite are homogeneous isotropic elastic materials, characterized by two elastic constants: Young's modulus $\mathrm{E}$ and Poisson ratio $v$ (or the Lamé coefficients: $\lambda$ and $\mu$ ). We denote: $E_{1}, v_{1}-$ the elastic constants of matrix material (sand) and $E_{2}$, $v_{2}$-the elastic constants of inclusions (sintered clay).

\subsection{Formulation of the homogenization problem}

In the process of homogenization three coordinates systems are introduced:

$\boldsymbol{X}\left(X_{1}, X_{2}, X_{3}\right)$ - dimensional coordinate system

$\boldsymbol{x}\left(x_{1}, x_{2}, x_{3}\right)=\boldsymbol{X}\left(X_{1}, X_{2}, X_{3}\right) / L$ - macroscopic coordinate system

$\boldsymbol{y}\left(y_{1}, y_{2}, y_{3}\right)=\boldsymbol{X}\left(X_{1}, X_{2}, X_{3}\right) / l$ - microscopic coordinate system

where $l$ is the characteristic microscopic length (here: dimension of the period) and $\mathrm{L}$ is the characteristic macroscopic length (here: dimension of the triaxial 
sample). The ratio $l / L=\varepsilon$ defines the scale separation parameter. We assume that the scale separation conditions are satisfied: $\mathcal{E}<<1$ (Auriault, 1991).

The equilibrium equation at the microscopic scale when inertia and body forces can be neglected, is written:

$$
\frac{\partial \sigma_{i j}}{\partial X_{j}}=0 \text { in } \Omega
$$

together with the Hooke's law:

$$
\sigma_{i j}=C_{i j k l} \varepsilon_{k l}(\boldsymbol{u})
$$

where the rigidity tensor $C_{i j k l}(\mathrm{y})$ is spatially dependent. The tensor $\varepsilon_{k l}=1 / 2\left(u_{k, l}+u_{l, k}\right)$ is the microscopic small deformation tensor.

At the interface $\Gamma$ (Figure 6) the continuity conditions of displacements and surface loads, are verified:

$$
[\boldsymbol{u}]_{\Gamma}=0 \text { and } \quad\left\lfloor\sigma_{i j} N_{j}\right\rfloor_{\Gamma}=0
$$

These conditions indicate that there is no jump of displacements and surface loads at the boundary $\Gamma$. In the asymptotic expansion homogenization method the homogenization problem has to be formulated using non-dimensions quantities. Note that we assumed that the properties of both constituents in all directions are of the same order of magnitude with respect to the scale separation parameter $\mathcal{E}$. Therefore, we can rewrite the problem [4] -[5] in the non-dimensional form

$$
\begin{aligned}
& \frac{\partial \sigma_{i j}}{\partial y_{j}}=0 \text { in } \Omega \\
& \text { with } \sigma_{i j}=C_{i j k l} \varepsilon_{k l}(\boldsymbol{u})
\end{aligned}
$$

In [6]-[7] we use the same notations for the non-dimensional displacements, stresses and rigidity tensor as for the dimensional quantities in [4]-[5].

In the next step, the unknown displacement is represented in a series expansion form:

$$
\boldsymbol{u}(\boldsymbol{x}, \boldsymbol{y})=\boldsymbol{u}^{(0)}(\boldsymbol{x}, \boldsymbol{y})+\varepsilon^{1} \boldsymbol{u}^{(1)}(\boldsymbol{x}, \boldsymbol{y})+\varepsilon^{2} \boldsymbol{u}^{(2)}(\boldsymbol{x}, \boldsymbol{y})+\ldots
$$

where all successive order terms are periodic functions.

Therefore, we have also: 


$$
\begin{aligned}
& \boldsymbol{\varepsilon}(x, y)=\varepsilon^{(0)}(x, y)+\varepsilon^{1} \varepsilon^{(1)}(x, y)+\varepsilon^{2} \varepsilon^{(2)}(x, y)+\ldots \\
& \sigma(x, y)=\sigma^{(0)}(x, y)+\varepsilon^{1} \sigma^{(1)}(x, y)+\varepsilon^{2} \sigma^{(2)}(x, y)+\ldots
\end{aligned}
$$

As usual in the two scale homogenization the derivative operator is defined as

$$
\frac{d}{d y_{i}}=\frac{\partial}{\partial y_{i}}+\varepsilon \frac{\partial}{\partial x_{i}}
$$

\subsection{Homogenization}

By applying the classical asymptotic expansion homogenization method to the problem [6]-[7], we can obtain the following results for the displacement function at successive orders (Sanchez-Palencia, 1980), (Bensoussan et al., 1978):

$$
\begin{aligned}
& u_{i}^{(0)}(\boldsymbol{x}, \boldsymbol{y})=u_{i}^{(0)}(\boldsymbol{x}) \\
& u_{i}^{(1)}(\boldsymbol{x}, \boldsymbol{y})=\xi_{i}^{k h} \varepsilon_{x k h}\left(\boldsymbol{u}^{(0)}\right)+\bar{u}_{i}^{(1)}(\boldsymbol{x})
\end{aligned}
$$

where $\xi_{i}^{k h}(\boldsymbol{y})$ is a particular periodic solution of the elasticity problem corresponding to the unit macroscopic strain tensor $\mathbf{E}^{\mathrm{kh}}=\left(e_{k} \otimes e_{h}+e_{h} \otimes e_{k}\right) / 2$. where $\mathrm{k}, \mathrm{h}=1,2,3$. The symbol $\otimes$ means the tensorial product between the unit vectors of the basis. This solution should also verify the zero volume average condition. The function $\bar{u}_{i}^{(1)}(\boldsymbol{x})$ is arbitrary.

At the next order homogenization of the problem [6]-[7] the macroscopic description is found as in (Sanchez-Palencia, 1980), (Bensoussan et al., 1978):

$$
\frac{\partial<\sigma_{i j}^{(0)}>}{\partial x_{j}}=0
$$

where the macroscopic stress tensor is defined as a volumetric average:

$$
<\sigma_{i j}^{(0)}>=\frac{1}{|\Omega|} \int_{\Omega} \sigma_{i j}^{(0)} d \Omega
$$

The macroscopic constitutive equation is obtained in the form: 


$$
<\sigma_{i j}^{(0)}>=C_{i j k h}^{*} \varepsilon_{x k h}\left(\boldsymbol{u}^{(0)}\right)
$$

where the macroscopic (effective) rigidity tensor is defined as:

$$
C_{i j k h}^{*}=<C_{i j k h}+C_{i j l m} \varepsilon_{y l m}\left(\xi^{k h}\right)>
$$

The tensors $\boldsymbol{\varepsilon}_{x}$ in [14] and $\boldsymbol{\varepsilon}_{y}$ in [15] are the macroscopic and the microscopic strain tensor, respectively.

As it can be seen from [12]-[15] the homogenization process provides us with the macroscopic description of the behaviour of the composite in form of the macroscopic Hooke's law in which the macroscopic rigidity tensor is a function of the elastic parameters of the components i.e. the microscopic rigidity tensors and the microstructure of the medium.

\section{Numerical computations of the rigidity tensor of the double porosity composite material}

\subsection{Local boundary value problem}

The rigidity tensor of the double porosity material can be computed from the solution of the local boundary value problem obtained from homogenization (Sanchez-Palencia, 1980), (Bensoussan et al., 1978):

$$
\begin{aligned}
& \frac{\partial}{\partial y_{j}}\left(C_{i j k h}\left(\varepsilon_{y k h}\left(\boldsymbol{u}^{(1)}\right)+\varepsilon_{x k h}\left(\boldsymbol{u}^{(0)}\right)\right)\right)=0 \text { in } \Omega \\
& \left\lfloor C_{i j k h}\left(\varepsilon_{y k h}\left(\boldsymbol{u}^{(1)}\right)+\varepsilon_{x k h}\left(\boldsymbol{u}^{(0)}\right)\right) N_{j}\right\rfloor_{\Gamma}=0 \\
& \left.\mid \boldsymbol{u}^{(1)}\right\rfloor_{\Gamma}=0
\end{aligned}
$$

where the first-order displacement field $\boldsymbol{u}^{(1)}$ is defined by equation [11]. Therefore, the unknown in the problem [16]-[17] is the periodic displacement field $\boldsymbol{\xi}$. The problem [16]-[17] can also be written in the form:

$$
\frac{\partial}{\partial y_{j}}\left(C_{i j l m} \varepsilon_{y l m}\left(\xi^{k h}\right)+C_{i j k h}\right)=0 \text { in } \Omega
$$




$$
\begin{aligned}
& \left|\left(C_{i j l m} \varepsilon_{y l m}\left(\xi^{k h}\right)+C_{i j k h}\right) N_{j}\right|_{\Gamma}=0 \\
& \left|\xi^{k h}\right|_{\Gamma}=0
\end{aligned}
$$

The problem [16]-[17] (or [18]-[101) has to be colved six times by imposing six unit macroscopic strain tensors $\mathbf{E}^{\mathrm{kh}}=\left(e_{k} \otimes e_{h}+e_{h} \otimes e_{k}\right) / 2$.

Then, the effective rigidity tensor $C_{i j k h}^{*}$ can be obtained by calculating the volume integral [15] based on homogenization. Using the engineering notations the fourth order tensor $C_{i j k h}^{*}$ can be converted into the second order tensor $D_{i j}^{*}$ by applying the usual conversion rule (Bacon and Pouyet, 2000, page 107). According to the above procedure the complete tensor $C_{i j k h}^{*}$ (and $D_{i j}^{*}$ ) can be determined, if the microstructure of the medium is known.

\subsection{Numerical results}

In this section the full macroscopic rigidity tensor for the microstructure of the composite geomaterial presented in Figure 1, is computed. The problem [16]-[17] issue from homogenization was solved taking into account the elasticity parameters of sand determined in the triaxial tests, Table 1. Since these parameters are strainstress dependent, the macroscopic rigidity tensor was calculated three times (for each unloading). On the contrary, the elastic parameters of sintered clay material were taken from the literature for bricks: $\mathrm{E}=14 \mathrm{GPa}$ and $v=0.2$ ( constant values). In order to obtain the full tensor $(6 \times 6$ components $)$ the problem [16]-[17] has to be solved (for each couple of the local elastic parameters) six times.

Since the microstructure possesses three symmetry planes (Figure 6), the material can be considered as orthotropic with 9 independent coefficients. The general form of the macroscopic tensor is (Berthelot, 1992): 


$$
\boldsymbol{D}^{*}=\left|\begin{array}{llllll}
D_{11} & D_{12} & D_{13} & 0 & 0 & 0 \\
D_{12} & D_{22} & D_{23} & 0 & 0 & 0 \\
D_{13} & D_{23} & D_{33} & 0 & 0 & 0 \\
0 & 0 & 0 & D_{44} & 0 & 0 \\
0 & 0 & 0 & 0 & D_{55} & 0 \\
0 & 0 & 0 & 0 & 0 & D_{66}
\end{array}\right|
$$

In the numerical computations we used the commercial finite elements code Comsol Multiphysics.

The three macroscopic rigidity tensors computed independently for three ranges of the axial strain are given in Annex 2. In particular, the following values of the component $D_{33}^{*}$ were obtained:

- For the axial strain range: $0.0128-0.0140$, we get $D_{33}^{*}=484.9 \mathrm{MPa}$

- For the axial strain range: $0.0177-0.0192$ we get $D_{33}^{*}=615.7 \mathrm{MPa}$

- For the axial strain range: $0.0230-0.0246$ we get $D_{33}^{*}=656.9 \mathrm{MPa}$

In Annex 2 we can observe that the rigidity tensor possesses the structure of an orthotropic tensor. The small values of the non-zero components like $D_{41}^{*}, D_{51}^{*}$, $D_{61}^{*}$ can be considered as numerical errors of approximation. It can also be seen that the terms $D_{11}^{*}$ and $D_{22}^{*}$ are approximately equal. Also, the terms $D_{44}^{*}$ and $D_{55}^{*}$ are very close to each other. This can be an indication of the state close to transversal isotropy with respect to the axis 3 (z). However, there is no full transversal isotropy.

As an example, in Figures 7 and 8 the displacement vector $\boldsymbol{\xi}$ corresponding to the case of unit macroscopic traction strain in the direction $\mathrm{z}$ (or 3) with the periodicity conditions, is presented. We can observe the three dimensional solution of the local boundary value problem (18)-(19). In Fig.7 the z-component, while in Fig. 8 the x-component, of the vector $\xi$ corresponding to the case of unit macroscopic strain in the $\mathrm{z}$ direction, are presented. The displacement field (the displacement corrector) is periodic in all directions which means that it takes the 
same values on the opposite external walls of the period. We can also see the zones of the maximum gradients (see the contribution in the definition [15]).

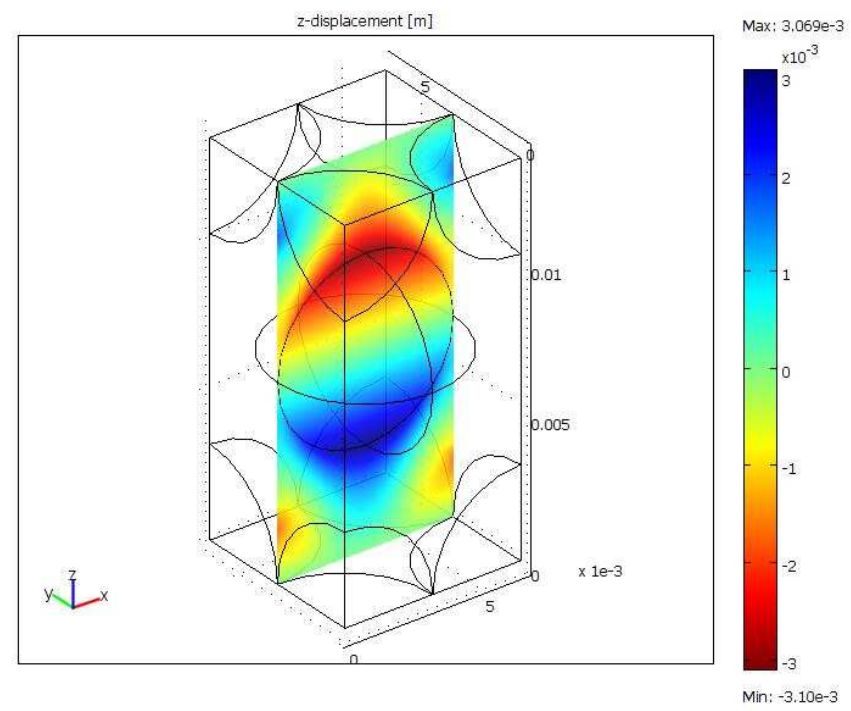

Figure 7 The z-component of the displacement field $\boldsymbol{\xi}$ for the case of unit macroscopic gradient in the $z$ direction

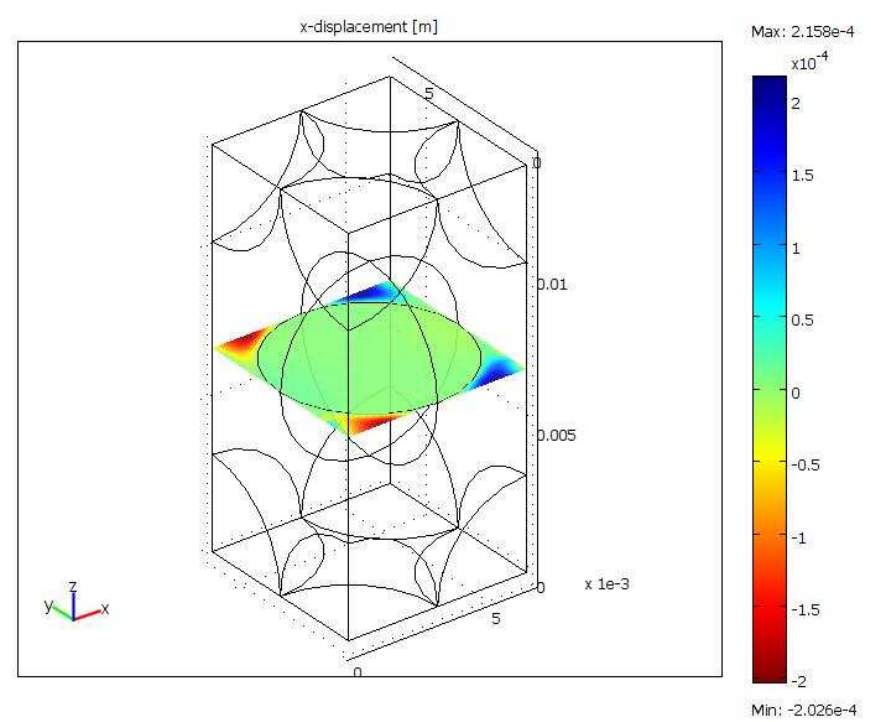

Figure 8 The $x$-component of the displacement field $\xi$ for the case of unit macroscopic gradient in the $z$ direction 


\subsection{Interpretation and comparison with the experimental results}

The results of the numerical calculations of the rigidity tensor presented in section 4.2 can be compared with the results of experiments summarized in section 2.3.2. The comparison concerns only two elasticity parameters determined from triaxial tests: the Young modulus in the direction $3\left(E_{3}\right)$ and the Poisson ratio $v$, Table 1. According to the theory of elasticity for orthotropic media the component $D_{33}^{*}$ of the rigidity tensor can be expressed as (Berthelot, 1992.)

$$
D_{33}^{*}=\frac{1-v_{12} v_{21}}{E_{1} E_{2} \Delta}
$$

where the coefficient $\Delta$ is expressed as a function of nine elastic parameters of orthotropy:

$$
\Delta=\frac{1-v_{12} v_{21}-v_{23} v_{32}-v_{31} v_{13}-2 v_{21} v_{32} v_{13}}{E_{1} E_{2} E_{3}}
$$

Further, as an approximation it will be assumed that the Poisson ratio of the double porosity medium is isotropic, which means that we can write:

$$
D_{33}^{*}=\frac{1-v^{2}}{1-3 v^{2}-2 v^{3}} E_{3}
$$

The relation [22] makes it possible to calculate the Young modulus $E_{3}$ for each calculated value of $D_{33}^{*}$ by considering that the Poisson ratio of the double porosity composite is known. Then, the value of $\mathrm{E}_{3}$ calculated from homogenization can be directly compared with the experimental value given in Table 1.

The interpretation was carried out in two steps:

i) In the first interpretation the measured value of the Poisson ratio of the composite was used in relation [22]

The results are given in Table 2. As we can see the macroscopic Young modulus shows important discrepancy between the calculated (296.1 MPa ,182.25MPa ,109.69MPa) and the measured values (286.11MPa $362.72 \mathrm{MPa}, 323.08 \mathrm{MPa}$ ). It is believed that the origin of this discrepancy is the Poisson ratio determined from the triaxial apparatus. It has to be pointed out the calculations proved to be very sensitive to the value of the Poisson ratio. This value is based on the measurement of the volume of water drained out of the medium. As we know, in the case of double porosity media there exists a memory effect in the water drainage, due to the low permeability of the second porosity ((Lewandowska et al. 2005), (Szymkiewicz et al., 2008), (Tran Ngoc at al., 
2011).). Therefore, the standard triaxial measurement conditions seem to be not appropriate in this case.

ii) In the second interpretation the value of the Poisson ratio of the composite used in the relation [22] was evaluated based on homogenization results and experimental data.

We calculated the average value of the coefficient related to the Poisson ratio namely $\delta=\left(1-v^{2}\right) /\left(1-3 v^{2}-2 v^{3}\right) \quad$ ([22]), using the ratios $D_{33}^{*} / E_{3}$ for each range of the strain-stress state, where $E_{3}$ is the measured Young modulus. The three values of the coefficient $1 / \delta$ are close to each other and equal respectively: 0.590 , $0.589,0.492$. The average value is $1 / \delta=0.557$. The corresponding Poisson ratio is equal to 0.374 . This value seems physically more realistic and was used in the comparison presented in Table 2 .

Table 2. Comparison between computed and measured values of Young modulus

\begin{tabular}{|c|c|c|c|c|c|}
\hline \multirow[b]{2}{*}{$\begin{array}{c}\text { Range of axial } \\
\text { strain }\end{array}$} & \multicolumn{2}{|c|}{ First interpretation } & \multicolumn{2}{|c|}{ Second interpretation } & \multirow[b]{2}{*}{$\begin{array}{c}\text { Measured } \\
\text { values of } E_{3} \\
{[\mathrm{MPa}]}\end{array}$} \\
\hline & $\begin{array}{c}\text { Poisson } \\
\text { ratio } v \\
\text { (measured) }\end{array}$ & $\begin{array}{c}\mathrm{E}_{3} \\
\text { (computed) } \\
{[\mathrm{MPa}]}\end{array}$ & $\begin{array}{c}\text { Poisson ratio } v \\
\text { obtained from the } \\
\text { ratio } \\
\mathrm{D}_{33} \text { (computed) } / \mathrm{E}_{3} \\
\text { (measured) }\end{array}$ & $\begin{array}{c}\mathrm{E}_{3} \\
\text { (computed) } \\
{[\mathrm{MPa}]}\end{array}$ & \\
\hline $0.0128-0.0140$ & 0.39 & 296.11 & 0.374 & 270.11 & 286.11 \\
\hline $0.0177-0.0192$ & 0.46 & 186.25 & 0.374 & 342.94 & 362.72 \\
\hline $0.0230-0.0246$ & 0.48 & 109.69 & 0.374 & 365.93 & 323.08 \\
\hline
\end{tabular}

Now, we obtain the following values for the Young modulus: 270.11MPa, 342.94MPa, 365.93MPa to be compared with 286.11MPa, 362.72MPa , 323.08MPa. It can be seen that the differences between the calculated and measured values are of the order of $10 \%$ in all cases Therefore, it can be concluded that good agreement was found. 


\section{Conclusions}

In this paper we presented the homogenization approach, based on the asymptotic expansion homogenization method, in order to compute the effective elasticity properties of a composite geomaterial (double porosity). The homogenization process was focused on the passage from the scale of the porous components to the scale of the composite.

A part of numerical simulations of the macroscopic rigidity tensor was validated through the experimental tests in the triaxial apparatus using a model periodic composite of anisotropic microstructure. The comparison showed good agreement between the computed and measured values of the Young modulus of the composite. To our knowledge very few validations of homogenization with experiments have been performed so far, especially for granular materials.

Some limitations of classical triaxial testing of highly heterogeneous porous media are discussed. In particular, the technique of measurement of volume changes based on the volume of water drained out of from the medium seems not appropriate in this case. Therefore, more experimental studies, using more advanced techniques, are postulated.

Finally, it can be concluded that asymptotic expansion homogenization is an excellent method of modelling physical phenomena in media with periodic microstructure and a useful technique to study the mechanical behaviour of composite geomaterials.

\section{Acknowledgements}

The experiments presented in this paper were conducted by Filip Grinke in the framework of his Master thesis during his stay in 2007 at the Laboratory Sols, Solides, Structures - Risques (3S-R) in Grenoble, France. The numerical calculations shown in this paper were done by Maciej Pilawski in 2011 as a part of his Master thesis at the Laboratory LMGC in Montpellier, France.

The first author would like to acknowledge the scientific advises and the technical assistance provided by the Laboratory Sols, Solides, Structures - Risques (3S-R) in Grenoble, France when performing the triaxial experiments. Special thanks are addressed to Pascal Charrier.

The financial support provided by European Erasmus program to both Master students (Silesian University of Technology), is greatly acknowledged. 


\section{Annex 1}

The first column of the tensor $\boldsymbol{D}^{*}$ is obtained by solving the problem [16]-[17] with periodic conditions for $\boldsymbol{\xi}$ and with unit macroscopic strain in the direction 1 . We have:

$$
\begin{aligned}
& D_{11}^{*}=\left(\lambda_{1}+\mu_{1}\right)\left(\phi_{1}+<\varepsilon_{11}>_{\Omega_{1}}\right)+\lambda_{1}\left(<\varepsilon_{22}>_{\Omega_{1}}+<\varepsilon_{33}>_{\Omega_{1}}\right)+ \\
& \left(\lambda_{2}+\mu_{2}\right)\left(\phi_{2}+<\varepsilon_{11}>_{\Omega_{2}}\right)+\lambda_{2}\left(<\varepsilon_{22}>_{\Omega_{2}}+<\varepsilon_{33}>_{\Omega_{2}}\right) \\
& D_{21}^{*}=\lambda_{1}\left(\phi_{1}+<\varepsilon_{11}>_{\Omega_{1}}+<\varepsilon_{33}>_{\Omega_{1}}\right)+\left(\lambda_{1}+\mu_{1}\right)<\varepsilon_{22}>_{\Omega_{1}}+ \\
& \left.\lambda_{2}\left(\phi_{2}+<\varepsilon_{11}>_{\Omega_{2}}+<\varepsilon_{33}>_{\Omega_{2}}\right)+\left(\lambda_{2}+\mu_{2}\right)<\varepsilon_{22}>_{\Omega_{2}}\right)+ \\
& D_{31}^{*}=\lambda_{1}\left(\phi_{1}+<\varepsilon_{11}>_{\Omega_{1}}+<\varepsilon_{22}>_{\Omega_{1}}\right)+\left(\lambda_{1}+\mu_{1}\right)<\varepsilon_{33}>_{\Omega_{1}}+ \\
& \lambda_{2}\left(\phi_{2}+<\varepsilon_{11}>_{\Omega_{2}}+<\varepsilon_{22}>_{\Omega_{2}}\right)+\left(\lambda_{2}+\mu_{2}\right)<\varepsilon_{33}>_{\Omega_{2}} \\
& D_{41}^{*}=2 \mu_{1}<\varepsilon_{23}>_{\Omega_{1}}+2 \mu_{2}<\varepsilon_{23}>_{\Omega_{2}} \\
& D_{51}^{*}=2 \mu_{1}<\varepsilon_{13}>_{\Omega_{1}}+2 \mu_{2}<\varepsilon_{13}>_{\Omega_{2}} \\
& D_{61}^{*}=2 \mu_{1}<\varepsilon_{12}>_{\Omega_{1}}+2 \mu_{2}<\varepsilon_{12}>_{\Omega_{2}}
\end{aligned}
$$

where:

$<\cdot>_{\Omega_{1}}=\frac{1}{|\Omega|} \int_{\Omega_{1}} \cdot d \Omega \quad \quad<\cdot>_{\Omega_{2}}=\frac{1}{|\Omega|} \int_{\Omega_{2}} \cdot d \Omega$ $\phi_{1}=\frac{\left|\Omega_{1}\right|}{|\Omega|}$ is the volumetric fraction of the material 1

$\phi_{2}=\frac{\left|\Omega_{2}\right|}{|\Omega|}$ is the volumetric fraction of the material 2 


\section{Annex 2}

The three macroscopic rigidity tensors for three ranges of axial strain are respectively:

- $\quad$ For the axial strain range: $0.0128-0.0140$

with : $\mathrm{E}_{1}=119.94 \mathrm{MPa}, v_{1}=0.35, \lambda_{1}=103.7 \mathrm{MPa}, \mu_{1}=44.42 \mathrm{MPa}$

$$
\boldsymbol{D}^{*}=\left|\begin{array}{rrrrrc}
1498.8 & 220.5 & 193.2 & -0.5 & -0.6 & 0.2 \\
220.5 & 1539.6 & 192.3 & -0.9 & -0.3 & 3 \\
193.2 & 192.3 & \mathbf{4 8 4 . 9} & -0.7 & -0.1 & 0.3 \\
-0.2 & -0.4 & 0.3 & 2616.9 & -0.2 & 0.2 \\
-0.3 & -0.1 & -0.1 & -0.2 & 2614.6 & -0.6 \\
0.1 & 1.5 & 1.5 & 0.2 & -0.6 & 2065.4
\end{array}\right| \mathrm{MPa}
$$

- $\quad$ For the axial strain range: $0.0177-0.0192$

with: $\mathrm{E}_{1}=163.67 \mathrm{MPa}, v_{1}=0.33, \lambda_{1}=119.44 \mathrm{MPa}, \mu_{1}=61.53 \mathrm{MPa}$

$$
\boldsymbol{D}^{*}=\left|\begin{array}{rrrrrr}
1678.7 & 254.5 & 226.6 & -0.6 & -0.6 & 0.2 \\
254.5 & 1715.8 & 225.5 & -1.0 & -0.3 & 2.9 \\
226.6 & 225.5 & \mathbf{6 1 5 . 7} & -0.8 & -0.1 & 0.4 \\
-0.3 & -0.5 & -0.4 & 2518.5 & -0.2 & 0.2 \\
-0.3 & -0.1 & -0.1 & -0.2 & 2516.1 & -0.7 \\
0.1 & 1.5 & 0.2 & 0.2 & -0.7 & 1972.8
\end{array}\right| \mathrm{MPa}
$$

- For the axial strain range: 0.0230- 0.0246

with: $\mathrm{E}_{1}=174.99 \mathrm{MPa}, v_{1}=0.33, \lambda_{1}=128 \mathrm{MPa}, \mu_{1}=65.8 \mathrm{MPa}$

$$
\boldsymbol{D}^{*}=\left|\begin{array}{rrrrrr}
1733.9 & 269.4 & 241.7 & -0.6 & -0.8 & 0.2 \\
269.4 & 1769.8 & 240.6 & -1.0 & -1.1 & 2.9 \\
241.7 & 240.6 & \mathbf{6 5 6 . 9} & -0.8 & -0.4 & 0.4 \\
-0.3 & -0.5 & -0.4 & 2493.9 & -0.2 & 0.3 \\
-0.3 & -0.2 & -0.1 & -0.2 & 2491.4 & -0.8 \\
0.1 & 1.5 & 0.2 & 0.3 & -0.8 & 1950.0
\end{array}\right| M P a
$$




\section{Bibliographie}

Auriault J.L., "Heterogeneous medium. Is an equivalent description possible?" Int. Journal Engng. Sci., vol. 29, No 7, 1991, p. 785-795.

Bacon C., Pouyet J., Mécanique des solides défromables, Hermes Sciences Publications, 2000.

Benssoussan A., Lions J.L., Papanicolaou G., Asymptotic Analysis for Periodic Structures, North-Holland, Amsterdam, Netherlands, 1978.

Berthelot J.-M., Matériaux composites, Paris Milan Barcelone Bonn MASSON, 1992.

Bornert M., Bretheau T., Gilormini P. Homogénisation en mécanique des matériaux 1. Matériaux aléatoire élastiques et milieux périodiques. Hermes Science Europe Ltd, 2001

Chatzigeorgiou G., Efendiev E., Lagoudas D. C. "Homogenization of aligned "fuzzy fibers" composite", International Journal of Solids and Structures, vol. 48, 2011, p. 2668-2680.

Desrues J., Orestis G.I. “ An Investigation of Diffuse Failure Modes in Undrained Triaxial Tests on Loose Sand, Soils and Foundations vol.46 No 5, 2006, p. 587-596.

François D., Pineau A., Zaoui A., Comportement mécanique des matériaux. Elasticité et plasticité, Hermes 2009.

Grinke F., "Modelling of elastic parameters using the upscaling approach", Master thesis, Silesian University of Technology, 2007. (in Polish)

Hall S., Bornert M., Desrues J., Pannier Y., Lenoir N., Viggiani G., Bésuelle P. "Discrete and Continuum analysis of localised deformation in sand using X-ray micro CT and Volumetric Digital Image Correlation”, Géotechnique vol.60 No 5, 2010, p. 315-322.

Lenoir N., Bornert M., Desrues J. , Bésuelle P., Viggiani G. "Volumetric Digital Image Correlation Applied to X-ray Microtomography Images from Triaxial Compression Tests on Argillaceous Rock “, Strain Vol. 43 Issue 3 2007, p. 193-205.

Lewandowska J., Szymkiewicz A., Gorczewska W., Vauclin M., "Infiltration in a doubleporosity soil: Experiments and comparison with a theoretical model", Water Resources Research, vol. 41, W02022, 2005, p. 1-14.

Lewandowska, J., Tran Ngoc, T. D., Vauclin M., Bertin H. "Water drainage in doubleporosity soils: experiments and micro-macro modelling", ASCE Journal of Geotechnical and Geoenvironmental Engineering, vol. 134(2), 2008, p. 231-243.

Pilawski M. "Modelling of chemo-mechanical couplings in soils: application to geological storage of CO2", Master thesis, Silesian University of Technology, 2011.

Sanchez-Palencia E., Non-Homogeneous Media and Vibration Theory, Berlin Heidelberg New York, Springer-Verlag, 1980.

Suquet P., "Effective properties of nonlinear composites". In Continuum Micromechanics. P. Suquet, Editor, CISM Lecture Notes vol. 377, Springer-Verlag, Berlin, 1997. 
Szymkiewicz A., Lewandowska J., Angulo-Jaramillo R., Butlanska J., "Two-scale modeling of unsaturated water flow in double porosity medium under axisymmetric conditions", Canadian Geotechnical Journal, vol. 45, 2008, p. 238-251.

Torquato S., Random heterogeneous materials. Microstructure and macroscopic properties. Springer, 2005

Tran Ngoc, T. D., Lewandowska, J., Vauclin M., Bertin H., "Two-scale modeling of dispersion in double porosity unsaturated media: homogenization and experiments", Int. J. Numer. Anal. Meth. Geomech. Vol. 35, 2011, p. 1536-1559.

REACH http://echa.europa.eu/reach_en.asp 